\title{
Precision COVID-19 Vaccine with Companion Diagnostics
}

\author{
Julianna Lisziewicz ${ }^{1}$ and Franco Lori \\ Research Institute for Genetic and Human Therapy, Bethesda, MD, and Milan, Italy
}

Submitted: April 3, 2020

Accepted: April 9, 2020

Published: April 10, 2020

\section{Abstract}

Although disease enhancement by antibodies has been described for corona and other viruses, nobody can predict whether such antibodies induced by the vaccine will not be harmful, especially after reinfection with a different strain. Alternative vaccines could induce memory T-cell responses, in the absence of antibodies, to kill newly infected cells. Preclinical testing of such precision vaccines is performed in silico without animal experiments since epitopes predicted to bind to three HLA class I molecules of a subject activate cytotoxic T-cell response with more than $80 \%$ probability. Data science can be utilized to select the immunogenic vaccine peptides from the coronavirus replicase protein and estimate the immune response rate in an HLA-genotyped population. Employing accessible platform technologies, a set of precision vaccines could be co-developed with an HLA-genotype based companion diagnostic to identify the vaccine that most likely induces responses in the subject. The goal of precision vaccination is to convert the deadly COVID-19 into asymptomatic disease and to avoid the potential risk of disease enhancement.

\section{Keywords}

Personalized Coronavirus Vaccine, Cytotoxic T-cell (CTL), Antibody, Immune Responses, Companion Diagnostic, Prediction of Vaccine Responder, Herd Immunity

Quote this article as: Lisziewicz J, Lori F, Precision COVID-19 Vaccine with Companion Diagnostics, Precis. Nanomed. 2020 April;3(2):487-494, https://doi.org/10.33218/001c.12561

\section{Purpose and Rationale}

There is an urgent need to develop a vaccine that protects people against COVID-19 and from reinfection with an evolved coronavirus strain. Current vaccine candidates intend to protect against infection by induction of antibody responses. However, coronavirusspecific antibodies can potentially enhance the disease, a risk that cannot be taken with evolving coronaviruses. An alternative approach is to protect people against the disease by vaccinating people with peptides that trigger coronavirus-specific memory $\mathrm{T}$-cell responses but cannot induce antibodies. Memory T-cells could patrol the lung for infected cells, and quickly proliferate to kill virus-infected cells before they start virus production. Matching vaccines epitopes and HLA genotype of a subject or a population optimizes immunogenicity and ensures herd immunity.

\section{Introduction}

At the end of 2019, only three months ago, we did not know COVID-19. In January 2020 the Chinese authorities responded to the outbreak with the same regulation that was introduced 1518 in London to stop the plague. That time bells ringing during burials reminded people to follow the prevention rules. Today governments introduced strict prevention rules but the coronavirus $(\mathrm{CoV})$ is spreading, and so far, we've lost about 100,000 lives in countries having the most developed healthcare systems in the world. At present (April 10, 2020), neither a specific antiviral nor a vaccine is available to combat COVID-19, and the World Health Organization (WHO) warned physicians

${ }^{1}$ Corresponding author: Julianna Lisziewicz, julianna.lisziewicz@icloud.com 
against the off-label use of medicines for the treatment of COVID-19. We can only hope that the pandemic will be slowed down by the 500year-old control measures and can prevent the death of about 40 million people [1].

This is not the first deadly human $\mathrm{CoV}$ epidemic. In 2002, 2004 and 2012, the world has experienced localized outbreaks caused by the same class of CoVs [2]. In January 2020 WHO experts forecasted that the COVID-19 would be another localized epidemic in China. In February 2020, we learned that it had become a deadly global pandemic. In the absence of effective drugs or a vaccine, the virus may have become more virulent following human-to-human transmission [3] and may cause a second wave of an epidemic since the number of people infected who might now be immune to the disease remains low. The differences among CoVs causing the COVID19 pandemic and the earlier outbreaks suggest that under selective pressure in humans or animals, CoVs evolve and leading to additional outbreaks accompanied by high death rates [4].

President Trump issued a "STAY HOME" order to mitigate the health and economic consequences of the COVID-19 pandemic and called for a vaccine that protects people against COVID-19 to be ready by the US election on November 2, 2020. Consequently, vaccine candidates have been produced within weeks after the publication of the first sequence data by Chinese scientists [5] and we expect to have safety and immunogenicity data in a small number of healthy volunteers by the summer. Such accelerated pre-clinical vaccine development was possible due to improved platform technologies tested in different indications.

\section{Discussion}

\section{Concerns and examples of Disease}

\section{Enhancement by Vaccine-Induced}

\section{Antibodies}

The immunological objective of the current prophylactic vaccine candidates is to induce neutralizing antibodies that inhibit the $\mathrm{CoV}$ entry to the cells [6]. The goal is to vaccinate approximately $60 \%$ of the population and to reach herd immunity against COVID-19 [7]. SARS-CoV-2 is not a highly mutagenic virus because it carries proof-reading machinery, like other $\mathrm{CoVs}$ [8]. Its relatively stable genome represents an advantage for vaccine development compared to highly mutating viruses like HIV-1. Nonetheless, strain variations occur during the evolution of $\mathrm{CoVs}$ that should be addressed during vaccine design.

Some of the current vaccine candidates will likely succeed in inducing neutralizing antibodies by either delivering or expressing viral proteins from mRNA or DNA in the body. These antibodies bind to the membrane (M), envelope (E) or spike (S) proteins of coronavirus and inhibit viral entry. However, similar approaches did not produce safe and effective vaccines against several viral diseases, including HIV-1, influenza, dengue, RSV (respiratory syncytial virus) and SARS$\mathrm{CoV}$. Independently from the platform technology used for vaccine development, several candidates induced neutralizing antibody responses against viral proteins but failed in either safety or efficacy.

One of the main safety concerns with the vaccine-induced virus-specific antibody responses was that they did not completely neutralize the virus. Instead, virus-specific antibodies guided viruses to enter those cells which they could not infect in the absence of antibodies (e.g., Fc receptor-expressing cells like macrophages and B-cells), causing an ordinarily mild infection to become deadly. This phenomenon led to troubling disease enhancement [9].

Enhancement of disease by the Dengue virus vaccine

Dengue virus infection is predominantly asymptomatic but can also cause severe disease, including hemorrhagic fever and shock syndrome. Primary infection induces monoclonal antibody-mediated protective immunity against reinfection by the same strain. The concern is that disease enhancement occurring after reinfections with other Dengue strain and among vaccine recipients having suboptimal or waning neutralizing antibody responses [10,11].

An alternative hypothesis for disease enhancement was that cytotoxic T-cell (CTL) and not antibody responses lead to impaired clearance of variant viruses infecting the same individual [12]. Specifically, memory T-cells induced by primary infection become 
predominant during reinfection with a heterologous strain (original antigenic sin), and these T-cells cannot control viral replication but produce an excessive amount of inflammatory cytokines (cytokine storm). Recent studies contradicted CTL mediated disease enhancements and provided proof that crossreactive memory CTLs capable of controlling viral replication [13]. Specifically, Dengue virus-specific memory CTLs patrol the skin for infected cells. In the case of reinfection, memory cells quickly proliferate and express cytotoxic molecules to kill virus-infected cells presenting the same epitope by matching HLA molecules [14]. Such virus-specific CTLs could not cause disease enhancement in Dengue vaccine recipients because Dengue vaccines failed to induce memory $\mathrm{T}$-cell responses.

\section{Enhancement of pneumonia by RSV vaccine} [9]

In 1963 an enhanced form of RSV disease was observed in 54 children during a vaccine trial that was initiated after showing protection in animal models. A total of $39 \%$ of vaccine recipients were infected with RSV and 18\% experienced the severe disease. In 1966, another RSV vaccine was administered to children in the United States that induced RSVspecific antibodies and no RSV-specific CTL responses. Vaccine-induced immune responses caused a "cytokine storm" and enhanced disease presenting with pneumonia that led to increased hospitalization rates and two deaths.

It is important to note that the systemic administration of RSV-neutralizing monoclonal antibody (palivizumab) prevents serious RSV-related respiratory disease in highrisk infants. These monoclonal antibodies could also enhance the disease in suboptimal concentration, but when administered in optimal concentration, the balance moves to complete neutralization of RSV [15].

\section{Enhancement of disease by experimental}

\section{CoV vaccines}

Most of the candidate $\mathrm{CoV}$ vaccines target the $\mathrm{S}$ protein because it is the most immunogenic antigen and can induce neutralizing antibodies enabling protection against infection in animal models [16]. However, in particular animal models, systemic administration of S-specific neutralizing antibodies accelerated disease course after infection instead of providing protection [17]. In one study, cats vaccinated against $\mathrm{CoV}$ became sicker than unvaccinated cats because virus-specific antibodies increased the virus uptake by macrophages [18]. In another study, vaccine candidates developed against SARS-CoV did not prevent MERS$\mathrm{CoV}$ infection, instead, animals experienced enhanced hepatitis, increased morbidity, and stronger inflammatory responses [19]. Virusspecific antibodies caused "cytokine storm" and triggered severe respiratory diseases not only by $\mathrm{CoV}$ but also by other highly pathogenic viruses like avian influenza and Spanish flu.

\section{The potential risk of vaccine-induced} enhancement of COVID

It is not known whether vaccine-induced antibodies could enhance the disease caused by SARS-CoV-2 infection or could mediate harmful immune responses during current or future pandemics. However, we cannot take the risk of a vaccine-induced enhanced disease progression during the second wave of the current epidemic or during another $\mathrm{CoV}$ epidemic. Since coronaviruses are constantly evolving [20,21], nobody can predict whether antibodies induced by mass vaccinations will not be harmful during future coronavirus outbreaks. A prophylactic vaccine that cannot be used in a large population because of a potential safety concern will not be useful to induce the herd immunity against COVID-19.

\section{Precision Vaccine to Induce Cytotoxic T-cells without Antibodies}

The immunological objective of a precision vaccine is to induce $\mathrm{CoV}$-specific CTL responses to kill infected cells in the absence of generating antibody responses. The goal of mass vaccination is to convert the epidemic to asymptomatic or mild disease that currently occurs in $81 \%$ of cases [22] and avoid the potential risk of disease enhancement.

Clinical studies with similar CoV (MERS, SARS) and HIV-1 suggest that vaccine-induced CTL responses might protect people from developing severe COVID-19. CoV infected patients with mild or asymptomatic disease rarely develop antibody responses. Instead, their disease is controlled by CoV-specific CTL responses. The more severe the illness, the greater the antibody response detected in patients in the convalescent phase [23]. In 1999 
we described the first HIV-infected patient, whose immune system controlled the virus replication after interruption of antiviral therapy. In his body, we demonstrated HIVspecific CTLs that could kill the HIV-infected cells in the absence of antibodies and thereby control the disease [24].

Vaccine-induced CTL might protect against $\mathrm{CoV}$ transmission if the CTL kills CoVinfected cells before starting the virus production. Such vaccine-induced CTL-S should target the first viral polyprotein expressed from ORF1ab of the viral RNA in the infected cells. The infected cell processes the newly synthesized polyprotein to peptides. Some of these peptides will be presented by HLA class I molecule on the surface of the infected cells that will be recognized by vaccine-induced CTLs. CTL targeting the replicase polyprotein will then kill infected cells before the cell start to synthesize the mRNAs needed for the structural viral proteins.

Matching Precision Vaccines with HLA genotype

To achieve herd immunity against COVID-19 the precision vaccine should induce CTL responses against the replicase polyprotein in approximately $60 \%$ of the population. CTL can be induced by peptides that bind to multiple HLA class I molecules in the body. Peptides binding to at least three HLA class I molecules in an individual can activate CTL response with more than $80 \%$ probability (Figure 1).

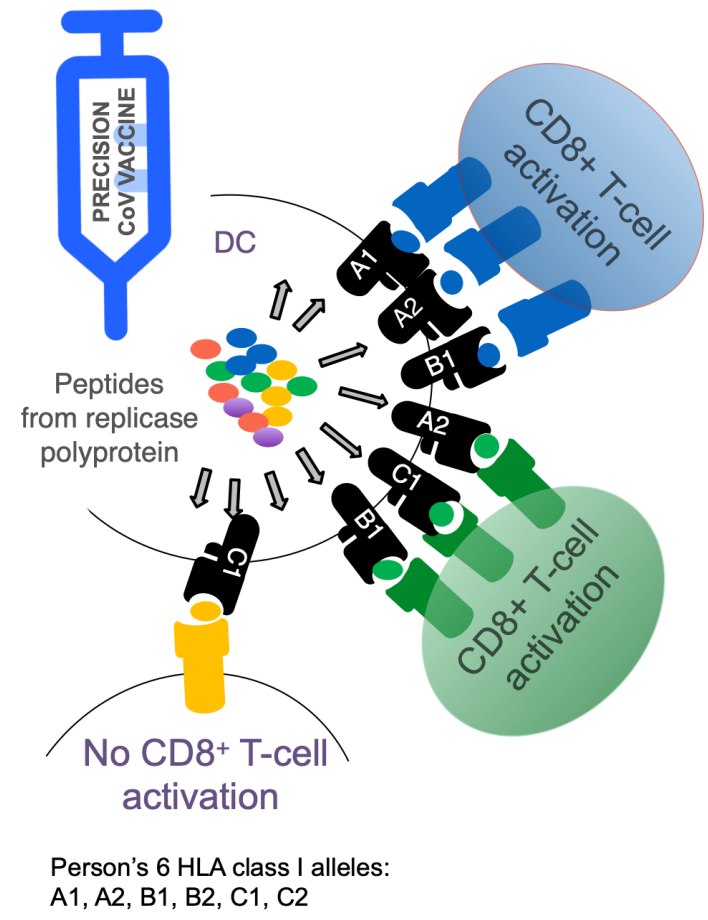

Figure 1. Induction of cytotoxic T-cell (CTL) responses by the precision corona virus (CoV) vaccine. The human population harbors 8-9 million HLA class I variants [31]. To trigger virus-specific CTL responses an epitope from CoV proteins must be presented by three of the 6 HLA class I molecules of a subject (HLA-A1, HLA-A2, HLA-B1, HLA-B2, HLA-C1, HLA-C2). Peptide vaccines containing a single HLA-binding epitope rarely activate CD8+CTLs. Instead, CD8+T-cells are typically triggered by peptides binding to at least three HLA class I molecules of a subject ( $J$ Lisziewicz, unpublished). Therefore, it is possible to accurately design peptides from the replicase proteins that induce CTL responses in an HLA genotyped subject. A peptide cocktail is suitable to induce herd immunity if CTL responses are predicted in at least $60 \%$ of the target population

The dominant three autologous HLA-binding peptides could be selected from the sequence data of the 7,096 amino acids long replicase polyprotein and HLA genotype data of the target population (available in transplantation databases). Data scientists could quickly design 
peptide cocktails that contain at least one peptide binding to 3 HLAs in each individual of the target population. Such a peptide cocktail will likely induce $\mathrm{CoV}$-specific CTL responses in $80 \%$ of the target population. This would be a "preferred" precision vaccine, a first-aid solution in the case of a fast-spreading virus such as SARS-CoV2 pandemic. However, the technology also lends itself to being applied as a personalized vaccine, in which case several peptide cocktail vaccines could be developed with a companion diagnostic test that predicts which vaccine induces the broadest CTL responses in an individual and most likely protects the subject from COVID-19. The companion diagnostic test would first determine the HLA genotype of the subject from a saliva sample, then would predict HLAbinding epitopes from the candidate vaccines (e.g., using the Immune Epitope Database epitope prediction tool at https://www.iedb.org) and last would determine for each available vaccine the peptides capable binding to 3 autologous HLA class I alleles and likely induce broad $\mathrm{CoV}$-specific CTLs. Accessible platform technologies could be used for rapid vaccine production: peptides with adjuvants, mRNA, DNA, etc. Similar precision peptide vaccines were previously used safely in cancer patients and induced potent CTL responses leading to tumor-killing [25]. More importantly, peptides derived from the replicase polyprotein cannot induce antibodies binding to $\mathrm{CoVs}$ and enhance disease progression in case people become infected.

Nanomaterial may be used to targeting the precision vaccine to antigen-presenting cells or the airway epithelial cells. Peptides and nucleic acids complexed with polyethylenimine (PEI) facilitate the induction of CTL responses. We have previously described that topical delivery of $100 \mathrm{~nm}$ PEIm/DNA nanoparticles targeted Langerhans that migrated to the lymph nodes and induced potent CTL responses in mice, money, and human subjects [26,27]. To direct the CTL responses to the lung, the similar nanoparticles could be targeted by inhalation to airway epithelial cells $[28,29]$. Besides PEI other polymeric nanoparticles can be used for delivering the vaccine peptides for induction of CTL potent responses [30].

\section{Conclusion}

We cannot run the risk that vaccine-induced antibodies will enhance the disease caused by coronaviruses during current or future pandemics. Conversely, we need to develop vaccines that induce precisely targeted CTL responses to kill cells infected with CoV. Such a prophylactic vaccine can be quickly developed and safely used in a large population of uninfected people to reach the desired herd immunity against COVID-19. Also, peptide cocktails in the precision vaccine can be quickly redesigned to combat future viral outbreaks.

\section{Conflict of Interests}

The authors declare no conflicts of interest. For signed statements, please contact the journal office: editor@precisionnanomedicine.com

Quote this article as Lisziewicz J, and Lori, F, Precision COVID-19 Vaccine with Companion Diagnostics, Precis. Nanomed. 2020;3(2):487-494, https://doi.org/10.33218/001c.12561

\section{Bibliography}

1. Graham F, Castelvecchi D, and Mallapaty S, Daily briefing: Coronavirus could have killed 40 million people if the world had not taken action, NATURE BRIEFING 31 MARCH 2020, www.nature.com/articles/d41586-020-00966-w

2. Hui, D. S. (2017). Epidemic and Emerging Coronaviruses (Severe Acute Respiratory Syndrome and Middle East Respiratory Syndrome). Clinics in Chest Medicine, 38(1), 71-86.

3. Zhao, G. (2007). SARS molecular epidemiology: a Chinese fairy tale of controlling an emerging zoonotic disease in the genomics era. Philosophical Transactions of the Royal Society B: Biological Sciences, 362(1482), 1063-1081.

4. Xu, J., Zhao, S., Teng, T., Abdalla, A. E., Zhu, W., Xie, L., ... Guo, X. (2020). Systematic Comparison of Two Animal-to-Human Transmitted Human Coronaviruses: SARS-CoV-2 and SARS-CoV. Viruses, 12(2), 244. 
5. Wu, F., Zhao, S., Yu, B., Chen, Y.-M., Wang, W., Song, Z.-G., Zhang, Y.-Z. (2020). A new coronavirus associated with human respiratory disease in China. Nature, 579(7798), 265-269. https://doi.org/10.1038/s41586-020-2008-3; https://www.nature.com/articles/s41586-020-2008-3

6. The pandemic pipeline; https://www.nature.com/articles/d41587-020-00005-Z

7. https://www.sciencemediacentre.org/expert-comments-about-herd-immunity/

8. Denison, M. R., Graham, R. L., Donaldson, E. F., Eckerle, L. D., \& Baric, R. S. (2011). Coronaviruses: an RNA proofreading machine regulates replication fidelity and diversity. RNA Biology, 8(2), 270-279

9. Smatti, M. K., Al Thani, A. A., \& Yassine, H. M. (2018). Viral-Induced Enhanced Disease Illness. Frontiers in Microbiology, 9, 2991

10. Sridhar, S., Luedtke, A., Langevin, E., Zhu, M., Bonaparte, M., Machabert, T., Savarino, S., Zambrano, B., Moureau, A., Khromava, A., Moodie, Z., Westling, T., Mascareñas, C., Frago, C., Cortés, M., Chansinghakul, D., Noriega, F., Bouckenooghe, A., Chen, J., DiazGranados, C. A. (2018). Effect of Dengue Serostatus on Dengue Vaccine Safety and Efficacy. New England Journal of Medicine, 379(4), 327-340.

11. de Silva, A. M., \& Harris, E. (2018). Which Dengue Vaccine Approach Is the Most Promising, and Should We Be Concerned about Enhanced Disease after Vaccination? The Path to a Dengue Vaccine: Learning from Human Natural Dengue Infection Studies and Vaccine Trials. Cold Spring Harbor Perspectives in Biology, 10(6),

12. Klenerman, P., \& Zinkernagel, R. M. (1998). Original antigenic sin impairs cytotoxic T lymphocyte responses to viruses bearing variant epitopes. Nature, 394(6692), 482-485.

13. Zehn, D., Turner, M. J., Lefrançois, L., \& Bevan, M. J. (2010). Lack of original antigenic sin in recall CD8(+) T cell responses. Journal of Immunology (Baltimore, MD): 1950), 184(11), 63206326.

14. Tian, Y., Grifoni, A., Sette, A., \& Weiskopf, D. (2019). Human T Cell Response to Dengue Virus Infection. Frontiers in Immunology (Vol. 10, p. 2125)

15. van Mechelen, L., Luytjes, W., de Haan, C. A. M., \& Wicht, O. (2016). RSV neutralization by palivizumab, but not by monoclonal antibodies targeting other epitopes, is augmented by Fc gamma receptors. Antiviral Research, 132, 1-5.

16. Aranda, S. S., \& Polack, F. P. (2019). Prevention of Pediatric Respiratory Syncytial Virus Lower Respiratory Tract Illness: Perspectives for the Next Decade. Frontiers in Immunology, 10, 1006. https://doi.org/10.3389/fimmu.2019.01006, https://www.ncbi.nlm.nih.gov/pmc/articles/PMC6524688/

17. Chen, J., and K. Subbarao. 2007. The immunobiology of SARS*. Annu. Rev. Immunol. 25: 443472.

18. Chris Ka-fai Li, Hao Wu, Huiping Yan, Shiwu Ma, Lili Wang, Mingxia Zhang, Xiaoping Tang, Nigel J. Temperton, Robin A. Weiss, Jason M. Brenchley, Daniel C. Douek, Juthathip Mongkolsapaya, Bac-Hai Tran, Chen-lung Steve Lin, Gavin R. Screaton, Jin-lin Hou, Andrew J. McMichael and Xiao-Ning Xu, T Cell Responses to Whole SARS Coronavirus in Humans, The Journal of Immunology, 181(8) 5490-5500 (2008); https://doi.org/10.4049/jimmunol.181.8.5490

19. H. Vennema et al., Early death after feline infectious peritonitis virus challenge due to recombinant vaccinia virus immunization. J. Virol. 64, 1407-1409 (1990).

20. Yong, C. Y., Ong, H. K., Yeap, S. K., Ho, K. L., \& Tan, W. S. (2019). Recent Advances in the Vaccine Development Against Middle East Respiratory Syndrome-Coronavirus. Frontiers in Microbiology, 10, 1781. https://doi.org/10.3389/fmicb.2019.01781

21. Wu, F., Zhao, S., Yu, B., Chen, Y.-M., Wang, W., Song, Z.-G., ... Zhang, Y.-Z. (2020). A new coronavirus associated with human respiratory disease in China. Nature, 579(7798), 265-269. https://doi.org/10.1038/s41586-020-2008-3

22. Cascella M, Rajnik M, Cuomo A, et al. Features, Evaluation and Treatment Coronavirus (COVID-19) [Updated 2020 Mar 20]. In: StatPearls [Internet]. Treasure Island (FL): StatPearls Publishing; 2020

23. Li, C. K., Wu, H., Yan, H., Ma, S., Wang, L., Zhang, M., Xu, X.-N. (2008). T Cell Responses to Whole SARS Coronavirus in Humans. The Journal of Immunology, 181(8), 5490 LP - 5500. 
24. Lisziewicz, J., Rosenberg, E., Lieberman, J., Jessen, H., Lopalco, L., Siliciano, R., Lori, F. (1999). Control of HIV despite the discontinuation of antiretroviral therapy [2]. New England Journal of Medicine, 340(21).

25. Fennemann, F. L., de Vries, I. J. M., Figdor, C. G., \& Verdoes, M. (2019). Attacking Tumors From All Sides: Personalized Multiplex Vaccines to Tackle Intratumor Heterogeneity. Frontiers in Immunology.

26. Lisziewicz, J., \& Toke, E. R. (2013). Nanomedicine applications towards the cure of HIV. Nanomedicine: Nanotechnology, Biology, and Medicine, 9(1).

27. Rodriguez, B., Asmuth, D. M., Matining, R. M., Spritzler, J., Jacobson, J. M., Mailliard, R. B., Li, X. D., Martinez, A. I., Tenorio, A. R., Lori, F., Lisziewicz, J., Yesmin, S., Rinaldo, C. R., \& Pollard, R. B. (2013). Safety, tolerability, and immunogenicity of repeated doses of dermavir, a candidate therapeutic HIV vaccine, in HIV-infected patients receiving combination antiretroviral therapy: results of the ACTG 5176 trial. Journal of Acquired Immune Deficiency Syndromes (1999), 64(4).

28. Di Gioia, S., \& Conese, M. (2009). Polyethylenimine-mediated gene delivery to the lung and therapeutic applications. Drug Design, Development and Therapy, 2, 163-188.

29. Kuzmov, A., \& Minko, T. (2015). Nanotechnology approaches for inhalation treatment of lung diseases. Journal of Controlled Release, 219, 500-518.

30. Luo, M., Wang, H., Wang, Z., Cai, H., Lu, Z., Li, Y., Du, M., Huang, G., Wang, C., Chen, X., Porembka, M. R., Lea, J., Frankel, A. E., Fu, Y.-X., Chen, Z. J., \& Gao, J. (2017). A STINGactivating nanovaccine for cancer immunotherapy. Nature Nanotechnology, 12(7), 648-654.

31. Robinson J, Guethlein LA, Cereb N, Yang SY, Norman PJ, Marsh SGE, et al. (2017) Distinguishing functional polymorphism from random variation in the sequences of $>10,000$ HLA-A, -B and -C alleles. PLoS Genet 13(6): e1006862. https://doi.org/10.1371/journal. pgen. 1006862 